\title{
Validity of a Novel Research-Grade Physical Activity and Sleep Monitor for Continuous Remote Patient Monitoring
}

\author{
Bríd McDevitt ${ }^{1, *(\mathbb{C}, \text { Lisa Moore }}{ }^{2}$, Nishat Akhtar ${ }^{1}$, James Connolly ${ }^{1, * \mathbb{C}}$, Rónán Doherty ${ }^{3} \mathbb{D}$ and William Scott ${ }^{2}$ \\ 1 Department of Computing, Letterkenny Institute of Technology, Letterkenny, F92 FC93 Donegal, Ireland; \\ L00138039@student.lyit.ie \\ 2 Department of Science, Letterkenny Institute of Technology, Letterkenny, F92 FC93 Donegal, Ireland; \\ L00115999@student.lyit.ie (L.M.); William.Scott@lyit.ie (W.S.) \\ 3 Department of Law \& Humanities, Letterkenny Institute of Technology, \\ Letterkenny, F92 FC93 Donegal, Ireland; Ronan.Doherty@lyit.ie \\ * Correspondence: Brid.McDevitt@lyit.ie (B.M.); James.Connolly@lyit.ie (J.C.)
}

Citation: McDevitt, B.; Moore, L.; Akhtar, N.; Connolly, J.; Doherty, R.; Scott, W. Validity of a Novel Research-Grade Physical Activity and Sleep Monitor for Continuous Remote Patient Monitoring. Sensors 2021, 21, 2034. https://doi.org/ $10.3390 / \mathrm{s} 21062034$

Academic Editor: Elfed Lewis

Received: 6 January 2021

Accepted: 9 March 2021

Published: 13 March 2021

Publisher's Note: MDPI stays neutral with regard to jurisdictional claims in published maps and institutional affiliations.

Copyright: (c) 2021 by the authors. Licensee MDPI, Basel, Switzerland. This article is an open access article distributed under the terms and conditions of the Creative Commons Attribution (CC BY) license (https:/ / creativecommons.org/licenses/by/ $4.0 /)$.

\begin{abstract}
In the midst of the COVID-19 pandemic, Remote Patient Monitoring technologies are highly important for clinicians and researchers. These connected-health technologies enable monitoring of patients and facilitate remote clinical trial research while reducing the potential for the spread of the novel coronavirus. There is a growing requirement for monitoring of the full $24 \mathrm{~h}$ spectrum of behaviours with a single research-grade sensor. This research describes a free-living and supervised protocol comparison study of the Verisense inertial measurement unit to assess physical activity and sleep parameters and compares it with the Actiwatch 2 actigraph. Fifteen adults (11 males, $23.4 \pm 3.4$ years and 4 females, $29 \pm 12.6$ years) wore both monitors for 2 consecutive days and nights in the free-living study while twelve adults (11 males, $23.4 \pm 3.4$ years and 1 female, $22 \pm 0$ years) wore both monitors for the duration of a gym-based supervised protocol study. Agreement of physical activity epoch-by-epoch data with activity classification of sedentary, light and moderate-to-vigorous activity and sleep metrics were evaluated using Spearman's rank-order correlation coefficients and Bland-Altman plots. For all activity, Verisense showed high agreement for both free-living and supervised protocol of $\mathrm{r}=0.85$ and $\mathrm{r}=0.78$, respectively. For physical activity classification, Verisense showed high agreement of sedentary activity of $r=0.72$ for free-living but low agreement of $r=0.36$ for supervised protocol; low agreement of light activity of $r=0.42$ for free-living and negligible agreement of $\mathrm{r}=-0.04$ for supervised protocol; and moderate agreement of moderate-to-vigorous activity of $\mathrm{r}=0.52$ for free-living with low agreement of $\mathrm{r}=0.49$ for supervised protocol. For sleep metrics, Verisense showed moderate agreement for sleep time and total sleep time of $r=0.66$ and 0.54 , respectively, but demonstrated high agreement for determination of wake time of $\mathrm{r}=0.83$. Overall, our results showed moderate-high agreement of Verisense with Actiwatch 2 for assessing epoch-by-epoch physical activity and sleep, but a lack of agreement for activity classifications. Future validation work of Verisense for activity cut-point potentially holds promise for $24 \mathrm{~h}$ continuous remote patient monitoring.
\end{abstract}

Keywords: actigraphy; accelerometer; inertial measurement unit; Digital Healthcare; free-living; supervised protocol; physical activity; activity cut-points; health behaviors; sleep monitoring

\section{Introduction}

In the era of ubiquitous digital connection, Remote Patient Monitoring (RPM) is an expanding and developing area of healthcare research and delivery improvement [1-3]. RPM technologies automatically monitor and report on patients' activity-related vital signs [4,5], oftentimes with chronic conditions [3,6]. In the midst of the COVID-19 pandemic, RPM technologies that enable contactless monitoring of patients are integral for minimising the spread of the novel coronavirus [7-9] while accommodating remote clinical research [10-12]. 
For human activity monitoring, physical behaviours that occur throughout a full $24 \mathrm{~h}$ day are categorised into physical activity (PA), sedentary behaviour (SB) and sleep [13]. These three behaviours are significant for research and health considerations due to their verifiable impact on health [14-16], both independently and symbiotically [17-19]. Time spent in one behaviour in a $24 \mathrm{~h}$ period will directly influence at least one of the other behaviours [19]. Higher sleep quality increases energy and reduces fatigue levels [20,21]. Reciprocally, greater PA ameliorates sleep quality $[20,22]$. Moreover, the optimal combination between time spent sleeping and in active behaviours (both light and moderate to vigorous physical activities (MVPA)) is associated with lower cardiovascular risk [23]. It is therefore advisable to target all behaviours together [24] in free-living observations of a $24 \mathrm{~h}$ day to better comprehend the individual and combined impacts of these activityrelated parameters [25].

Accelerometer-based monitors that balance cost and feasibility have emerged as valid tools to directly quantify movement [26] that results from PA [27], SB [28,29] and sleep [30,31]. Accelerometers offer low-cost continuous substitution for polysomnography (PSG) [32] and indirect calorimetry [33-35], which are the gold standards for sleep and PA monitoring. To date, studies that measured waking movement behaviour and sleep typically utilised two separate accelerometer models [36,37]. Given that waking activity behaviour and sleep can be directly assessed with similar approaches for body movement acceleration detection [38], a logical development for convenience and cost-effectiveness would be to utilise one single accelerometer that can measure PA and sleep over the full $24 \mathrm{~h}$ spectrum [22]. Ref. [39] reported the existence of only one recently developed research-specific device that fulfils such requirements: the Actigraph Link (ActiGraph, LLC), therefore advocating for more monitors that can objectively and simultaneously measure waking movement and sleep, and minimise the burden on research cohorts to wear distinct devices that measure behaviours independently [25,40].

The Actiwatch 2 accelerometer (Philips Respironics, Eindhoven, The Netherlands) is a commonly utilised wrist-worn sleep-monitor, that has been validated and widely used for detection of sleep duration and sleep quality [41]. The Actiwatch 2 also facilitates measurement of PA in proprietary activity counts per time unit. A study by [36] developed PA thresholds to segment sedentary, light and MVPA levels of activity for the Actiwatch 2 by comparing activity counts to indirect calorimetry using a portable metabolic cart and an actigraph device [42]. Another study [43] validated the Actiwatch 2 for PA by examination of activity level against energy expenditure measured using indirect calorimetry with the Actiwatch 2. Results were strongly correlated to a widely validated PA device, the ActiGraph wGT3X-BT, thus making the Actiwatch 2 a valid device for both PA and sleep monitoring [36]. This establishes the Actiwatch 2 as a device for the full spectrum of $24 \mathrm{~h}$ activity monitoring, which is desirable in clinical research involving participants where participant burden is a pertinent issue for both sleep and PA. This is supported by aforementioned findings based on ActiGraph wGT3X-BT, and other previous studies on its validity in sleep monitoring $[43,44]$ and thus, a suitable device for validation of novel sensors such as that presented in this research.

The Actiwatch 2 is relatively expensive (approximately US \$1500), and produces summative information with a requirement for manual data upload. A viable alternative research-grade accelerometer that can measure waking movement behaviour and sleep, that is more cost-effective, with access to raw sensor data and a long battery life with automated data upload to a secure cloud server, would be beneficial for long-term activity and sleep measurement and assessment. Recently, the use of the accelerometers that provide raw acceleration data in place of a proprietary filtered data units has increased [45,46], with a desired criterion being the production of temporal raw data, as is normally outputted from research-grade monitors [47]. Long-lasting battery life and memory storage is an important consideration to professionals who require high-resolution outputs. However, a necessary equilibrium is the production of detailed data without compromising other 
practical considerations such as sensor dimensions and burden on participants to wear the device [48].

This study implements Verisense, a novel wrist-worn inertial measurement unit (IMU) sensor designed for clinical trials, and developed by Shimmer Research Ltd. (Dublin, Ireland). Verisense accommodates continuous RPM through integration of their wearable sensor, base-station, and cloud platform for automatic data upload. Verisense outputs raw IMU sensor data on waking movement behaviour and sleep and has up to six months of battery life with no recharging. As discussed by [49], these functionalities fit the desirable requirements for sensor systems measuring healthcare parameters in that they uninterruptedly measure and wirelessly report all health-related information after one initial setup, placing minimal restrictions on participants for interaction or maintenance. While Verisense accommodates these requirements, Actiwatch 2 does not, demonstrating a need for Verisense to be validated for future studies. Other alternatives were considered; however, any sensors of similar specifications were either more expensive or lacked in at least one key feature that Verisense offered [50,51]. Furthermore, sensors that differed in body placement location such as shoe-worn devices were deemed unrealistic for the purposes of sleep monitoring as necessitated by the research [52,53], due to unfeasibility of wearing footwear while asleep. Wrist-worn placements were chosen for this study in keeping with the findings from a systematic review and practical considerations of device placement in [54], and the superiority for sleep quality-metrics from wrist-worn sensors as reported in [55]. Actiwatch 2 was selected for its reliability as a single device capable of $24 \mathrm{~h}$ activity and sleep monitoring and Verisense was chosen to investigate the potential match for that reliability while meeting the additional desirable functions for battery life, open-source algorithms and automatic data upload that is needed for further $24 \mathrm{~h} \mathrm{RPM} \mathrm{in}$ PA and sleep studies.

There are four aims of this study: (1) compare temporally matched PA measured via Verisense and Actiwatch 2 over the data collection period; (2) compare PA cut-points of sedentary, light and MVPA measured via Actiwatch 2 and Verisense over the data collection period; (3) evaluate the ability of Verisense to determine sleep time, wake time and total sleep time (TST) compared to sleep metrics measured by Actiwatch 2 and (4) evaluate the objective and subjective comparisons between sensor data and the participant diaries.

\section{Materials and Methods}

\subsection{Participants}

As previously reported in preliminary findings from Moore et al. [56], participants were recruited from the student population at Letterkenny Institute of Technology (LYIT) through word of mouth. Participants were required to satisfy the following inclusion criteria: (1) >18 years of age, (2) no self-reported condition(s) that could impede PA, and (3) no self-reported sleep issues. All participants were provided with information sheets that clearly defined the study protocols and objectives, and consent forms. Consenting participants were selected for inclusion for Free Living $(\mathrm{FL})(\mathrm{n}=20)$ and Supervised Protocol (SP) $(n=16)$. There were zero participant withdrawals from the study. Five FL data sets were excluded from analysis due to a malfunction of one or both of the wearable sensors. Four incomplete SP data sets were excluded from analysis as a result of COVID-19 lockdown restrictions in Ireland impeding the completion of the study. A total of 15 valid sets of FL data and 12 valid sets of SP data were obtained from the participants for statistical analysis. Each participant was allocated a random participant ID. The study was approved by the Research Ethics Committee at LYIT. Descriptive variables include self-reported sex and age as collected by a study researcher before study commencement. Body mass index was calculated using measured height and weight of participants $\left(\mathrm{kg} / \mathrm{m}^{2}\right)$.

\subsection{Instrumentation}

An Actigraph is a portable device that records accelerometry data at the wrist. The Actiwatch 2 (Phillips Respironics Mini-Mitter) is a lightweight (16 grams) actigraphy device 
$(43 \mathrm{~mm} \times 23 \mathrm{~mm} \times 10 \mathrm{~mm}$ as worn on wrist) that utilises a piezoelectric sensor to detect vertical accelerations spanning 0.5-2.0 g (Figure 1). The Actiwatch 2 has a rechargeable battery with a life of approximately 22 days at $15 \mathrm{~s}$ epochs and 30 days at $60 \mathrm{~s}$ epochs with a sampling rate of $32 \mathrm{~Hz}$. Actiwatch 2 has a resistance rating of IPX7 meaning that it can be immersed in water for a maximum of $30 \mathrm{~min}$ between $15 \mathrm{~cm}$ to $1 \mathrm{~m}$ water depth [57]. Activity counts from the device are proprietary and reported peak acceleration is detected over each epoch to determine sleep and wake states [38]. Data are transferred from the devices to the Actiware software via USB docking station. $15 \mathrm{~s}$ epochs were used for data collection. The auto scoring setting on the Actiware software was used to determine sleep and wake from the Actiwatch 2 data and were used as the comparators.

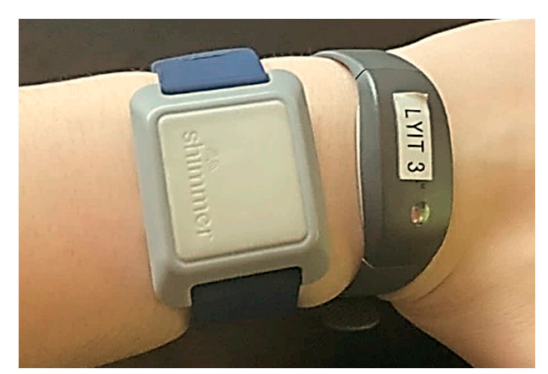

Figure 1. Verisense (left) and Actiwatch 2 (right) wrist-worn devices as worn by a participant.

Verisense is a wrist-worn IMU which records PA and sleep duration (Figure 1). The Verisense system (Shimmer Research Ltd.) consists of a tri-axial accelerometer and gyroscope (29.6 g) ( $35 \mathrm{~mm} \times 43 \mathrm{~mm} \times 12 \mathrm{~mm}$ as worn on wrist) with an accompanying base station and accompanying software application that transfers data locally to Shimmers remote cloud system. The Verisense IMU sensor is a CE certified, Class 1 medical device [58] that records the wearer's activity and sleep metrics onto an in-built flash memory, and once within range of the base station, data is automatically uploaded to the application on the base station. The base station then performs an automated data upload to an Amazon Web Services (AWS) server where data is then relayed to the relevant trial site and researchers have remote access to raw or analysed data for PA and sleep. Researchers can select the preferred sampling rate from the Verisense software with fixed values of $12.5 \mathrm{~Hz}, 25 \mathrm{~Hz}$, $50 \mathrm{~Hz}, 100 \mathrm{~Hz}, 200 \mathrm{~Hz}, 400 \mathrm{~Hz}, 800 \mathrm{~Hz}$ and $1600 \mathrm{~Hz}$. Verisense has a resistance rating of IP55 meaning that it is protected against water jets projected by a nozzle $(6.3 \mathrm{~mm})$ from any angle and is protected against dust that could interfere with the normal operation of the product but is not fully dust proof. The Verisense sensor has up to six months battery life with no need for recharging [59].

\subsection{Study Protocol under Free-Living Conditions}

As informed by previous research from [56] describing preliminary results, the validation study was divided into two testing sections: (1) a FL section and (2) a SP section. Each participant wore an Actiwatch 2 and Verisense sensors on the non-dominant wrist for the duration of both testing sections. Participants wore both sensors continuously for $48 \mathrm{~h}$, resulting in two nights of sleep, and two days of PA data. For the study duration, $48 \mathrm{~h}$ was defined as the period spanning between midday on the first day until midday on the third day of measurement. Participants were requested to complete an accompanying PA and sleep diary during the FL section which was custom adapted by the research team based on commonly used sleep [60] and PA diaries [61,62] utilised in research. The diary included questions regarding time in bed, time lights out, time woke up, time lights on, time out of bed and total hours of sedentary, light and MVPA activity for each $24 \mathrm{~h}$ period. All participants adhered to this request. The outcome measures were (1) temporally aligned in $15 \mathrm{~s}$ epochs from both devices for the $48 \mathrm{~h}$ FL and SP, (2) aligned with PA levels for FL and SP using thresholds located in the literature, and (3) sleep and wake metrics from both devices using the participant diary as a guide [30] and using an open-source 
heuristic algorithm referred to as Heuristic algorithm looking at Distribution of Change in Z-Angle (HDCZA), without any accompanying sleep log to guide as described in detail elsewhere [63]. Participants were asked to remove the devices for any water-based activities and to record periods of non-wear in the adapted diary. Sleep and activity data were retrieved from the Verisense Dashboard [64] and the Actiwatch 2 data was obtained using Philips Actiware (v 6.0.9) [65]. Both devices were initialised prior to study commencement with internal clocks automatically synchronised to a research team computer.

\subsection{Study Protocol under Supervised-Protocol Conditions}

Within the SP section, participants wore both the Actiwatch 2 and Verisense sensors on their non-dominant wrist for the duration of the gym-based activities shown in Table 1 and were supervised throughout by a study researcher. The walking and jogging activities were completed on treadmills and/or across a flat gym surface. Activities were completed in a randomised order as determined by study researchers. Participants were instructed by a study researcher when to start and stop each activity. The activity description, and exact start and finish times were recorded by the researcher. After each activity, participants were instructed to stand completely still with arms resting comfortably by the sides of the body for $60 \mathrm{~s}$ to accommodate a clear delineation between activity transitions in sensor outputs. The SP section was completed within a maximum of 45 mins, varying upon each participant's walking/jogging pace for self-paced activities. The activities included were informed by previous studies which recommended various sedentary and locomotor activities listed in Table 1 [66]. Other activities suggested for inclusion in validation studies, such as bicycling were excluded from this study due to feasibility constraints, however ascending and descending of steps as recommended by [67] were included as feasibility permitted in the gym facilities utilised.

Table 1. Supervised protocol activities.

\begin{tabular}{cc}
\hline Activity & Time (min) \\
\hline Sit & 2 \\
$3.5 \mathrm{~km} / \mathrm{h}$ walk on treadmill & 2 \\
$4.5 \mathrm{~km} / \mathrm{h}$ walk on treadmill & 2 \\
$5.5 \mathrm{~km} / \mathrm{h}$ walk on treadmill & 2 \\
$7.5 \mathrm{~km} / \mathrm{h}$ walk on treadmill & 2 \\
$11.5 \mathrm{~km} / \mathrm{h}$ walk on treadmill & 2 \\
Walking on flat surface at regular pace & 6 \\
$200 \mathrm{~m}$ slow walk on flat surface & \\
$200 \mathrm{~m}$ normal walk on flat surface & \\
$200 \mathrm{~m}$ fast walk on flat surface & \\
$200 \mathrm{~m}$ jog walk on flat surface & \\
Ascend 15 steps & \\
Descend 15 steps & \\
\hline
\end{tabular}

\subsection{Data Reduction}

Immediately following each participants test session, both sensors were removed from the participant, and the data were downloaded to a research team member's computer using relevant software (Actiware software v6.5.2 and Verisense Cloud Platform). Actiwatch 2 activity counts were presented in timestamped .csv format in 15-second epochs. Raw triaxial acceleration values from Verisense were converted into one omnidirectional measure of body acceleration. For this, the vector magnitude (VM) was taken from the three axes and then subtracted by the value of gravity (g) as in Equation (1), $\sqrt{ }\left(x^{2}+y^{2}+z^{2}\right)-1 g$, after which, negative values were rounded up to zero, referred to as Euclidean norm minus one (ENMO). 
ENMO, described in detail elsewhere $[27,68,69]$, where $1000=1000$ milli-gravitational units $=1$ gravitational unit, is defined as:

$$
r_{i}=\sqrt{x_{i}^{2}+y_{i}^{2}+z_{i}^{2}}=i^{t h}
$$

where: $i^{\text {th }}=$ vector magnitude at each time point.

For the computation of the ENMO metric, the resultant Verisense .csv files were combined into one .csv file using a custom script and values presented in $\mathrm{m} / \mathrm{s}^{2}$ were converted to $\mathrm{g}$ using the same custom script, then exported into $\mathrm{R}$ statistical software V3.1.2 [70] for processing using the GGIR package (V2.2) [30,63,68,69,71,72]. The ENMO method subtracts a fixed offset value of 1 gravitational unit at each time point to correct for gravity $[27,68,69]$. Negative ENMO values are rounded up to zero to reduce bias and error [27,69]. By design, the ENMO metric is sensitive to poor calibration [69]. Therefore, to address these calibration issues, ENMO was calculated using the GGIR package, which auto-calibrates the raw triaxial accelerometer signal [69]. The package regenerated the time-stamps and the files were exported into SPSS Statistical software V26 [73] for analysis. Further information on the accelerometer calibration technique can be found elsewhere [69]. ENMO was expressed in $\mathrm{mg}$ and calculated over $15 \mathrm{~s}$ epochs as previously described in preliminary findings from Moore et al. [56] to facilitate epoch-by-epoch comparison between sensors.

\subsection{Data Analysis}

Spearman's rank-order correlation coefficient $[74,75]$ was used to assess the temporal relationship between activity counts from the Actiwatch 2, and ENMO from Verisense because the data were not normally distributed. In accordance with a well-documented scale for medical research, we considered correlations of $0-0.3$ as negligible correlation, $0.3-0.5$ as low correlation, $0.5-0.7$ as moderate correlation, $0.7-0.9$ as high correlation, and $0.9-1.0$ as very high correlation. A $p<0.05$ was considered statistically significant for all analyses [76].

PA classification were compared between devices using sensitivity, specificity and overall accuracy [77] as well as Spearman's rank-order correlation coefficient. Sleep parameters were compared between each sensor with Spearman's rank-order correlation coefficient. To evaluate the agreement between two measurement methods [76], BlandAltman plots [78] were used to visually evaluate the agreement of the sleep summaries collected by the two sensors. This technique plots the difference score between two measures against their means. Sleep diary data were used to set the time in bed period. Actiwatch data were converted into "sleep" and "wake" using Actiware's proprietary algorithms, previously explained in [38]. To determine the occurrence of wake and sleep states in Verisense, the open-source GGIR algorithm was used to automatically determine sleep onset time and sleep offset time, as widely validated in previous research both with and without corresponding sleep diaries $[30,63,68,69,71,72]$.

\section{Results}

For the FL study, 15 participants were included (11 males, mean age ( \pm SD) $23( \pm 3.4)$ years, mean BMI $( \pm \mathrm{SD}) 23.9( \pm 2.6) \mathrm{kg} / \mathrm{m}^{2}$, and 4 females, mean age $( \pm \mathrm{SD}) 29( \pm 12.6)$, mean BMI $\left.22.6( \pm 1.3) \mathrm{kg} / \mathrm{m}^{2}\right)$. For the SP study, 12 participants were included (11 males, mean age $\left( \pm\right.$ SD) $23( \pm 3.4)$ years, mean BMI $\left( \pm\right.$ SD) $23.9( \pm 2.6) \mathrm{kg} / \mathrm{m}^{2}$, and 1 female, mean age $( \pm \mathrm{SD}) 22( \pm 0)$, mean BMI $\left.22.8( \pm 0) \mathrm{kg} / \mathrm{m}^{2}\right)$. Everyone who participated in the SP study also participated in the FL study. All participants were third-level students. Activities performed by the participants during the test days included sitting (e.g., at lectures), standing (e.g., practical classes) and walking. A number of participants were highly active (e.g., did workouts) whereas others were mainly sedentary during the test days.

The study compared epoch-by-epoch data obtained from both the Actiwatch 2 and Verisense devices over the $48 \mathrm{~h}$ FL study period from all 15 participants. The overall 
patterns observed between the Actiwatch 2 and Verisense visually appear to be quite similar for the $48 \mathrm{~h}$ FL study (Figures 2 and 3). Movement data from $48 \mathrm{~h}$ FL absolute activity for the Actiwatch 2 and Verisense sensors were highly correlated $(r=0.85 \pm 0.04$, range: $0.77-0.92, \mathrm{n}=15$; Spearman correlation).

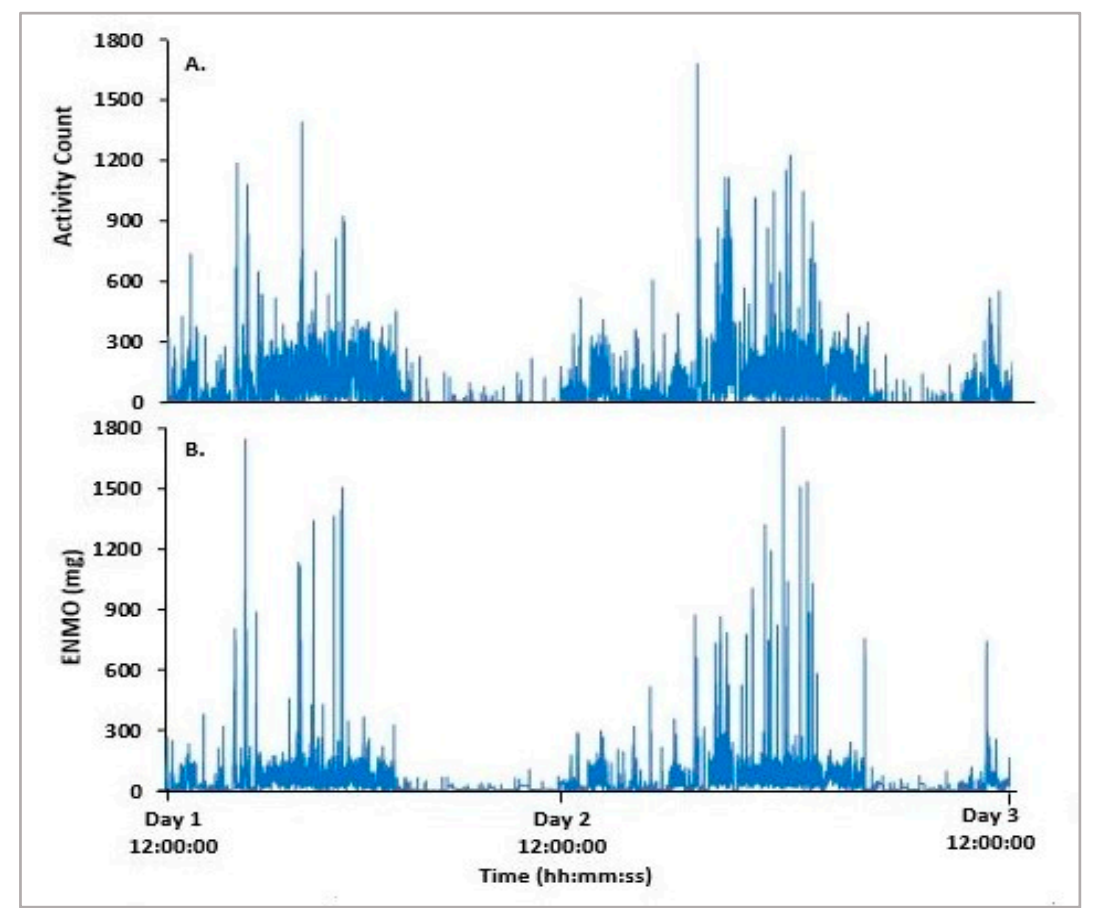

Figure 2. Representative epoch-by-epoch activity tracing of Actiwatch 2 (A) and Verisense (B) from a participant over a $48 \mathrm{~h}$ FL study.

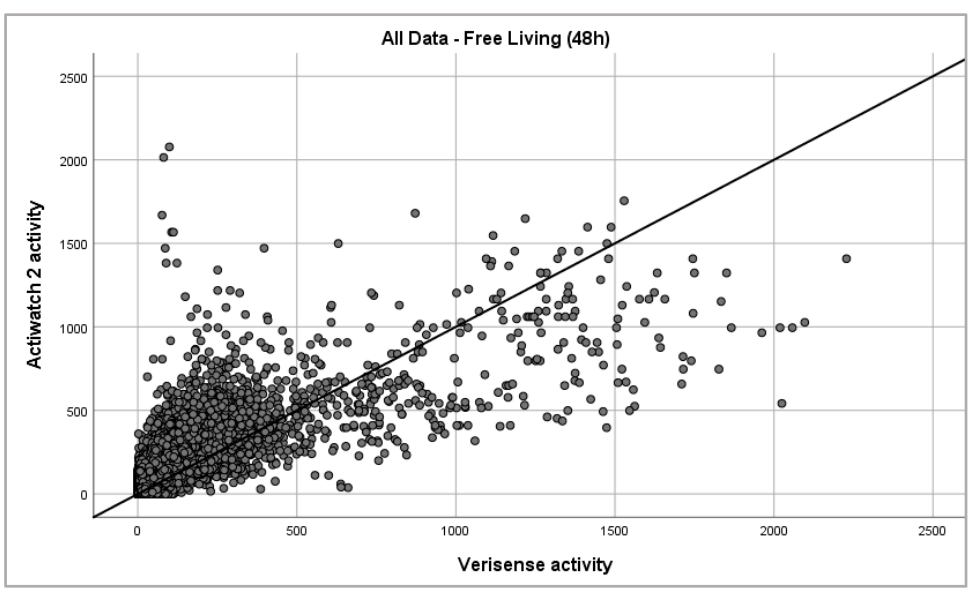

Figure 3. Epoch-by-epoch absolute activity of Actiwatch 2 and Verisense as recorded from all subjects over $48 \mathrm{~h}(163,759$ data points).

Epoch-by-epoch data obtained from both the Actiwatch 2 and Verisense sensors were compared over the gym-based SP from 12 participants. The overall patterns observed between the Actiwatch 2 and Verisense appear to be visually quite similar for the gymbased SP study (Figures 4 and 5). Within participants, gym-based SP activity for the Actiwatch 2 and Verisense sensors were also highly correlated $(r=0.78 \pm 0.05$, range: $0.72-0.88, \mathrm{n}=12$; Spearman correlation). 


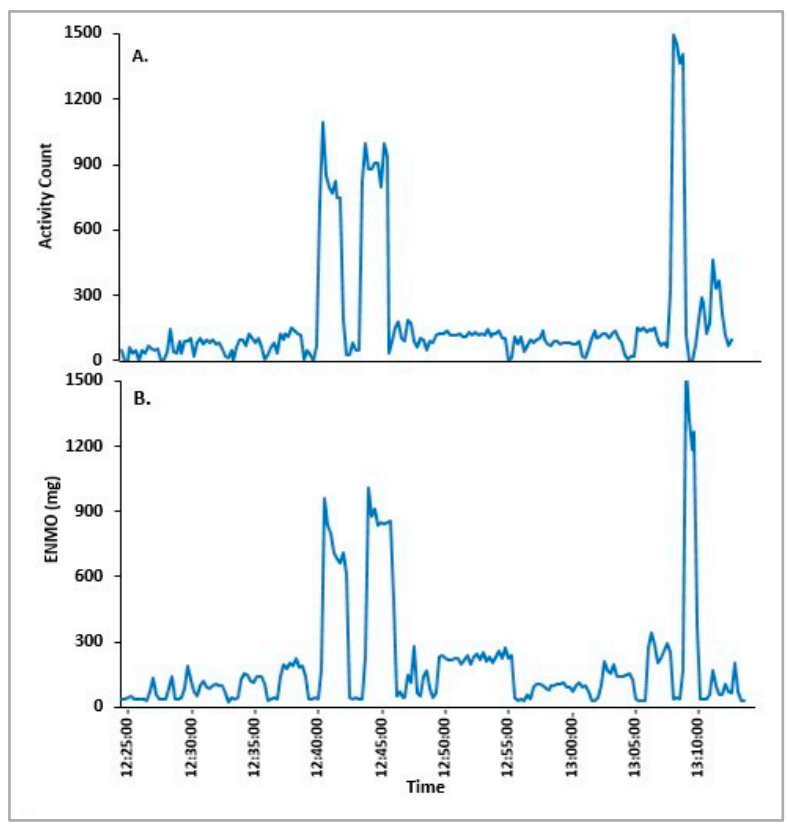

Figure 4. Representative epoch-by-epoch activity tracing of Actiwatch 2 (A) and Verisense (B) from a participant over a gym-based SP study.

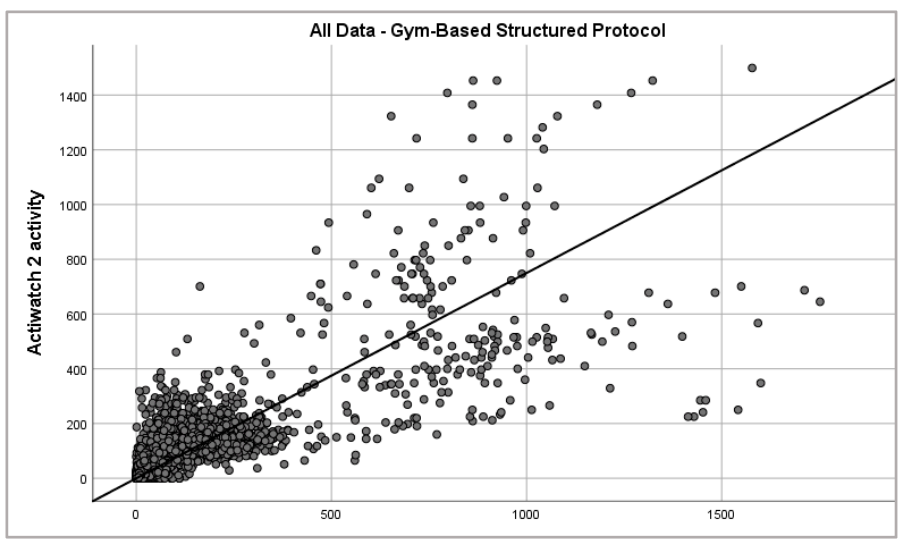

Figure 5. Epoch-by-epoch absolute activity of Actiwatch 2 and Verisense as recorded from all subjects over gym-based supervised protocol (2330 data points).

Epoch-by-epoch level data were segmented into PA levels of sedentary, light and MVPA activity using previously published cut-points from [36] for Actiwatch 2 . These cutpoints were defined as sedentary $<145$, light $<=274$, moderate $>274$ and vigorous $>=597$. However, limitations of sample size and female-only study participants is a notable limitation in the validity of application to these cut-points in other populations. For this reason, Actiwatch 2 cut-points could not be used as a gold-standard from which to base corresponding Verisense cut-points using Receiver Operating Characteristic (ROC) curves. All Verisense epoch-by-epoch level data were segmented using GGIR and processed in $\mathrm{R}$ following mean cut-points utilised by two similar specification accelerometers $[27,28]$ of sedentary $<45$, light $<=97$, moderate $>97$ and vigorous $>=423$. To date, no cut-point validation has occurred for Verisense, hence these cut-points should be noted as experimental only.

Using the aforementioned cut-point values, sensitivity, specificity and accuracy of the imputed PA levels were examined, as determined by the Actiwatch 2. For the most part, there was moderate correspondence in the determination of cut-points by Verisense and Actiwatch 2. The observed concordance between Verisense and Actiwatch 2 is presented in Table 2. 
Table 2. Overall accuracy, sensitivity and specificity comparative performance and Spearman's correlation coefficient and range values of Verisense in detecting PA levels during FL and SP, in comparison to Actiwatch 2.

\begin{tabular}{cccccccc}
\hline & & Overall Accuracy $\%$ * & Sensitivity \% * & Specificity \% * & Spearman's * & Range * $^{*}$ \\
\hline \multirow{2}{*}{ Sedentary } & FL & 92.9 & 93.2 & 90.9 & $0.72 \pm 0.05$ & $0.65-0.83$ \\
\cline { 2 - 7 } & SP & 56.0 & 35.6 & 96.4 & $0.36 \pm 0.016$ & $0.11-0.63$ \\
\hline \multirow{2}{*}{ Light } & FL & 90.4 & 51.3 & 93.9 & $0.42 \pm 0.06$ & $0.27-0.53$ \\
\cline { 2 - 8 } & SP & 65.2 & 14.4 & 79.9 & $-0.04 \pm 0.10$ & $-0.27-0.12$ \\
\hline \multirow{2}{*}{ MVPA } & FL & 95.8 & 84.8 & 96.0 & $0.52 \pm 0.09$ & $0.35-0.78$ \\
\cline { 2 - 8 } & SP & 65.0 & 95.2 & 63.1 & $0.49 \pm 0.13$ & $0.16-0.60$ \\
\hline
\end{tabular}

* Accuracy, sensitivity, specificity and Spearman's of Verisense as compared to Actiwatch 2

Within FL participants, cut-point generated activity for the Actiwatch 2 and Verisense sensors were highly correlated for sedentary, low correlated for light and moderately correlated for MVPA. Within SP participants, cut-point generated activity for the Actiwatch 2 and Verisense sensors were low correlated for sedentary, negligibly correlated for light and low correlated for MVPA as presented in Table 2.

Epoch-by-epoch sleep metrics were calculated from the two sensors using both the sleep/wake classification proprietary algorithm for Actiwatch 2 and GGIR for Verisense. Using both algorithms, TST (min), sleep and wake times were reported from both sensors. TST are the epochs scored as sleep within the reported time span between sleep and wake. The agreement of the three sleep indicators (TST, sleep time and wake time) between participant diaries, Actiwatch 2, Verisense guided by participant diary and Verisense unguided was tested using Spearman correlation coefficients and Bland- Altman plots. To facilitate comparison, the sleep start time and wake time were converted from time to numerical values for statistical comparison. values were calculated for time duration from 18:00 until sleep start time, and time values from 00:00 until wake time. All times were converted to mins. No day sleepers were included in the study.

Tables 3-5 shows the Spearman's correlation coefficient of sleep start times, wake times and TST for participant diaries, Actiwatch 2, Verisense guided by participant diary and Verisense unguided. First, the total average sleep start times of all the participants were $402.5 \pm 92.8 \mathrm{~min}, 417.4 \pm 149.5 \mathrm{~min}, 390.7 .5 \pm 127.4 \mathrm{~min}$ and $398.9 \pm 114.5 \mathrm{~min}$, respectively. Next, the total average wake times of all the participants were $555.2 \pm 96.7 \mathrm{~min}$, $575.97 \pm 115.8 \mathrm{~min}, 550.5 \pm 118.4 \mathrm{~min}$ and $576.9 \pm 118.8 \mathrm{~min}$, respectively. Finally, the total average sleep times of all the participants were $515.1 \pm 107.6 \mathrm{~min}, 524.8 \pm 131.8 \mathrm{~min}$, $526.6 \pm 121.5 \mathrm{~min}$ and $543.1 \pm 102.4 \mathrm{~min}$, respectively.

To examine the possibility of systematic bias in overall sleep parameter scoring, BlandAltman plots were generated to visually inspect the level of agreement between Verisense and Actiwatch 2 results (Figures 6-8). For sleep time, wake time and TST, the spread of the differences visually appears to be even, with no bias in overestimation or underestimation of sleep, wake or TST.

Table 3. Sleep correlations.

\begin{tabular}{ccccc}
\hline Sleep Time & Participant Diary & Actiwatch 2 & Verisense-Guided & Verisense-Unguided \\
\hline Participant Diary & - & 0.53 & 0.79 & 0.46 \\
\hline Actiwatch 2 & 0.53 & - & 0.66 & 0.43 \\
\hline Verisense-Guided & 0.79 & 0.66 & - & 0.67 \\
\hline Verisense-Unguided & 0.46 & 0.43 & 0.67 & - \\
\hline
\end{tabular}

$p<0.01$, correlation coefficient $(r=0.00-0.30$ : negligible correlation, $r=0.30-0.50$ : low correlation, $r=0.50-0.70$ : moderate correlation, $\mathrm{r}=0.70-0.90$ : high correlation, $\mathrm{r}=0.90-1.00$ : very high correlation) 
Table 4. Wake correlations.

\begin{tabular}{ccccc}
\hline Wake Time & Participant Diary & Actiwatch 2 & Verisense-Guided & Verisense-Unguided \\
\hline Participant Diary & - & 0.89 & 0.90 & 0.80 \\
\hline Actiwatch 2 & 0.89 & - & 0.83 & 0.80 \\
\hline Verisense-Guided & 0.90 & 0.83 & - & 0.82 \\
\hline Verisense-Unguided & 0.80 & 0.80 & 0.82 & - \\
\hline
\end{tabular}

$p<0.01$, correlation coefficient $(\mathrm{r}=0.00-0.30$ : negligible correlation, $\mathrm{r}=0.30-0.50$ : low correlation, $\mathrm{r}=0.50-0.70$ : moderate correlation, $\mathrm{r}=0.70-0.90$ : high correlation, $\mathrm{r}=0.90-1.00$ : very high correlation).

Table 5. TST correlations.

\begin{tabular}{ccccc}
\hline Total Sleep Time & Participant Diary & Actiwatch 2 & Verisense-Guided & Verisense-Unguided \\
\hline Participant Diary & - & 0.46 & 0.75 & 0.44 \\
\hline Actiwatch 2 & 0.46 & - & 0.54 & 0.31 \\
\hline Verisense-Guided & 0.75 & 0.54 & - & 0.63 \\
\hline Verisense-Unguided & 0.44 & 0.31 & 0.63 & - \\
\hline
\end{tabular}

$p<0.01$, correlation coefficient $(\mathrm{r}=0.00-0.30$ : negligible correlation, $\mathrm{r}=0.30-0.50$ : low correlation, $\mathrm{r}=0.50-0.70$ : moderate correlation, $\mathrm{r}=0.70-0.90$ : high correlation, $\mathrm{r}=0.90-1.00$ : very high correlation).

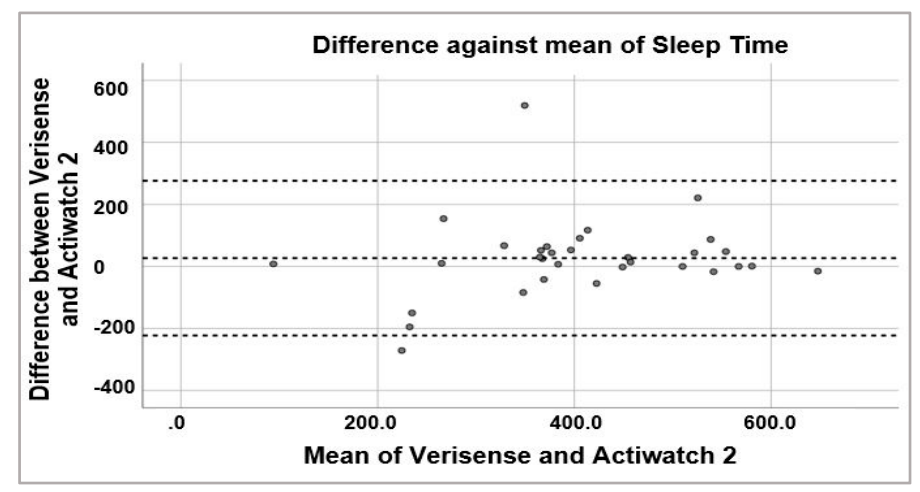

Figure 6. Bland-Altman plot of sleep times estimated by Verisense as compared to Actiwatch 2.

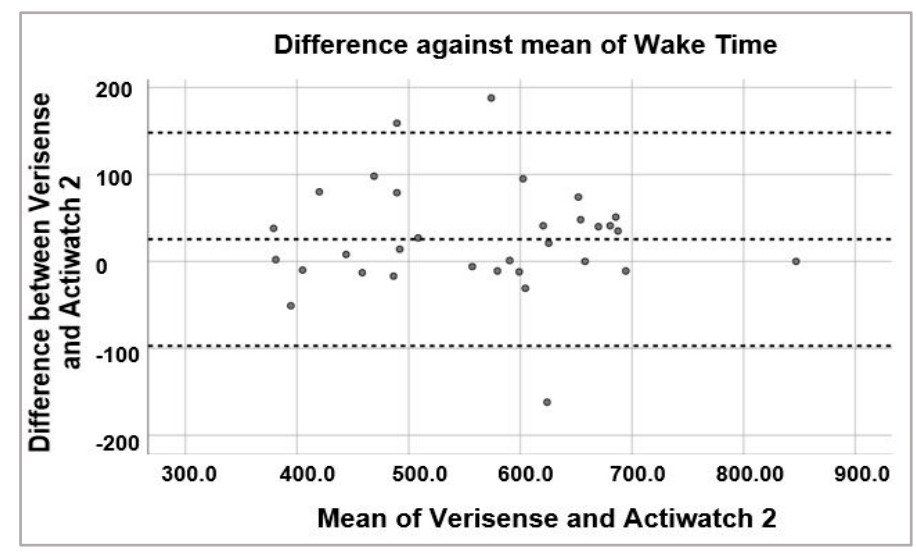

Figure 7. Bland-Altman plot of wake times estimated by Verisense as compared to Actiwatch 2. 


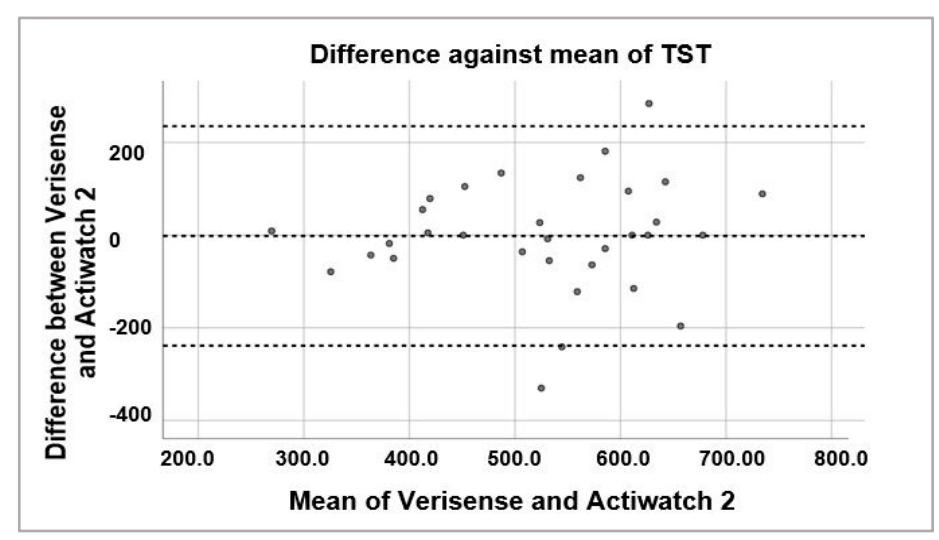

Figure 8. Bland-Altman plot of TST estimated by Verisense as compared to Actiwatch 2.

\section{Discussion}

To our knowledge, this is the first validation study where the Verisense IMU was compared to an actigraph for activity and sleep monitoring. In comparing the accuracy of Verisense, a novel research-grade wearable sensor, against a clinical/research-grade actigraphy device, Actiwatch 2, we find that the former device performs similarly in the estimation of epoch-by-epoch activity scoring and sleep parameters, although future studies on PA level classifications need further examination.

There are notable differences between the Verisense and the Actiwatch 2. While present on the Actiwatch 2, the Verisense lacks a light sensor, a feature often useful in identifying bed and wake times. The Actiwatch 2 stores data at a lower average resolution (e.g., $15 \mathrm{~s}$ and $30 \mathrm{~s}$ epochs at $32 \mathrm{~Hz}$ ) in comparison to the Verisense which is capable of raw data monitoring and storage ranging up to $1600 \mathrm{~Hz}$, facilitating higher resolution data with potential for greater accuracy. Verisense devices also remotely uploads all data to a secure cloud portal, eliminating the need for participants to attend a research facility to have data from the device downloaded, which is necessary with the Actiwatch 2. Significantly, Verisense provides access to raw accelerometer data which in place of a proprietary unit such as activity counts and filtered data which is now a desired part of research and increasing in adoption [45]. Verisense also has a long-lasting battery life of up to 6 months with no need for recharging, while most devices designed for clinical trials require frequent recharging and manual data upload. For longer duration longitudinal studies, these attributes of Verisense could be of significant benefit.

The adoption of wearable technology in healthcare and clinical trials continues to increase, however the paucity of standards for sensor algorithms can hamper their utilisation in research [79]. To address this, a healthcare industry open-wearables initiative (OWEAR) has been established [80]. The initiative seeks to develop open source algorithms and software for wearable sensor data analysis available to all medical device and pharmaceutical companies in a pre-competitive environment as a service to the industry [79-81].

One key area of debate in accelerometry activity monitoring is most appropriate wear-site for maximum accuracy [82,83]. Both devices presented in the study were wristworn, however many previous studies have utilised sensors worn at the waist $[84,85]$. It is therefore imperative to understand whether wrist-worn devices are an acceptable alternative compared to the waist for PA monitoring. In 2011, the U.S. National Health and Nutrition Examination Survey began using wrist-worn accelerometers to estimate PA [86]. Ref. [48] reported that a waist-worn GENEA triaxial accelerometer produced an almost identical correlation with energy expenditure as the same model worn on the wrist. However, Ref. [87] reported that a uniaxial accelerometer worn on the wrist and hip of participants during lifestyle activities produced a discrepancy in variance in energy expenditure with the waist-worn accelerometer accounting for $31.7 \%$ of the variance and the wrist-worn accelerometer explaining only $3.3 \%$ of the variance. This suggests that a triaxial accelerometer such as Verisense is suitable as a wrist-worn wearable device for PA. 
Future studies will compare Verisense to indirect caliometry for further PA classification validation, as this is the true, current gold standard in determination of PA cut-points [33-35]. The current results do, however, support the potential use of Verisense as an actigraphy device for the purpose of activity and sleep monitoring.

\section{Conclusions}

Verisense, a novel research-grade wearable device, produces activity and sleep parameters that are comparable to a research-grade actigraph and thus, can be used as a PA monitor and an actigraph for sleep monitoring.

It is a pertinent challenge to find a single reliable sensor to assess both PA and sleep while adhering to budgets and maximising participant compliance through minimising burden. We assert that this work is a step forward in examining and validating one single novel research-grade wearable device that provides access to raw sensor data, automated upload and long-lasting battery life which all contribute to negating participant burden, especially for longitudinal research. Concomitant validation of the Actiwatch 2 and Verisense against indirect caliometry will be an important future step to determine full equivalence of PA classification.

Author Contributions: Conceptualization, B.M., L.M., N.A. and J.C.; methodology, B.M., L.M., N.A. and J.C.; software, B.M.; validation, L.M., B.M. and N.A.; formal analysis, B.M.; curation, B.M. and L.M.; writing—original draft preparation, L.M.; writing—review and editing, B.M.; visualization, B.M.; supervision, J.C., W.S. and R.D.; project administration, J.C., W.S. and R.D.; funding acquisition, J.C. All authors have read and agreed to the published version of the manuscript.

Funding: This research was funded by Letterkenny Institute of Technology.

Institutional Review Board Statement: The study was conducted according to the guidelines of the Declaration of Helsinki, and approved by the Ethics Committee of Letterkenny Institute of Technology (December 2019).

Informed Consent Statement: Informed consent was obtained from all subjects involved in the study.

Data Availability Statement: The datasets generated during and/or analysed during the current study are available from the corresponding author on reasonable request.

Conflicts of Interest: The authors declare no conflict of interest.

\section{Abbreviations}

The following abbreviations are used in this manuscript:

ENMO Euclidean Norm Minus One

FL Free-living

SP Supervised Protocol

TST Total Sleep Time

IMU Inertial Measurement Unit

SB Sedentary Behaviour

PA Physical Activity

PSG Polysomnography

\section{References}

1. Vegesna, A.; Tran, M.; Angelaccio, M.; Arcona, S. Remote Patient Monitoring via Non-Invasive Digital Technologies: A Systematic Review. Telemed. e-Health 2017, 23, 3-17. [CrossRef]

2. Seshadri, D.R.; Davies, E.V.; Harlow, E.R.; Hsu, J.J.; Knighton, S.C.; Walker, T.A.; Voos, J.E.; Drummond, C.K. Wearable Sensors for COVID-19: A Call to Action to Harness Our Digital Infrastructure for Remote Patient Monitoring and Virtual Assessments. Front. Digit. Health 2020, 2. [CrossRef]

3. Malasinghe, L.P.; Ramzan, N.; Dahal, K. Remote patient monitoring: A comprehensive study. J. Ambient Intell. Hum. Comput. 2019, 10, 57-76. [CrossRef]

4. Steinhubl, S.R.; Muse, E.D.; Topol, E.J. Can Mobile Health Technologies Transform Health Care? JAMA 2013, 310, $2395-2396$. [CrossRef] 
5. Steinhubl, S.R.; Muse, E.D.; Topol, E.J. The emerging field of mobile health. Sci. Transl. Med. 2015, 7, 283rv3. [CrossRef] [PubMed]

6. Hamine, S.; Gerth-Guyette, E.; Faulx, D.; Green, B.B.; Ginsburg, A.S. Impact of mHealth Chronic Disease Management on Treatment Adherence and Patient Outcomes: A Systematic Review. J. Med. Internet Res. 2015, 17, e52. [CrossRef] [PubMed]

7. Siwicki, B. A Guide to Connected Health Device and Remote Patient Monitoring Vendors. Healthcare IT Nezws, 6 May 2020. Available online: https:/ / www.healthcareitnews.com/news/guide-connected-health-device-and-remote-patient-monitoringvendors(accessed on 3 August 2020).

8. Smith, A.C.; Thomas, E.; Snoswell, C.L.; Haydon, H.; Mehrotra, A.; Clemensen, J.; Caffery, L.J. Telehealth for global emergencies: Implications for coronavirus disease 2019 (COVID-19). J. Telemed. Telecare 2020, 26, 309-313. [CrossRef]

9. Wosik, J.; Fudim, M.; Cameron, B.; Gellad, Z.F.; Cho, A.; Phinney, D.; Curtis, S.; Roman, M.; Poon, E.G.; Ferranti, J.; et al. Telehealth transformation: COVID-19 and the rise of virtual care. J. Am. Med. Inform. Assoc. 2020, 27, 957-962. [CrossRef] [PubMed]

10. Free, C.; Phillips, G.; Watson, L.; Galli, L.; Felix, L.; Edwards, P.; Patel, V.; Haines, A. The Effectiveness of Mobile-Health Technologies to Improve Health Care Service Delivery Processes: A Systematic Review and Meta-Analysis. PLoS Med. 2013, 10, e1001363. [CrossRef] [PubMed]

11. Wijesooriya, N.R.; Mishra, V.; Brand, P.L.; Rubin, B.K. COVID-19 and telehealth, education, and research adaptations. Paediatr. Respir. Rev. 2020, 35, 38-42. [CrossRef]

12. Gill, G. Wearables for Remote Patient Monitoring: How Do We Get There? 2020. Available online: https:/ digitalhealthbuzz. com/wearables-for-remote-patient-monitoring-how-do-we-get-there/ (accessed on 30 December 2020).

13. Migueles, J.H.; Cadenas-Sanchez, C.; Rowlands, A.V.; Henriksson, P.; Shiroma, E.J.; Acosta, F.M.; Rodriguez-Ayllon, M.; EstebanCornejo, I.; Plaza-Florido, A.; Gil-Cosano, J.J.; et al. Comparability of accelerometer signal aggregation metrics across placements and dominant wrist cut points for the assessment of physical activity in adults. Sci. Rep. 2019, 9. [CrossRef] [PubMed]

14. Lee, I.-M.; Shiroma, E.J.; Lobelo, F.; Puska, P.; Blair, S.N.; Katzmarzyk, P.T. Effect of physical inactivity on major non-communicable diseases worldwide: An analysis of burden of disease and life expectancy. Lancet 2012, 380, 219-229. [CrossRef]

15. Ekelund, U.; Steene-Johannessen, J.; Brown, W.J.; Fagerland, M.W.; Owen, N.; Powell, K.E.; Bauman, A.; Lee, I.-M. Does physical activity attenuate, or even eliminate, the detrimental association of sitting time with mortality? A harmonised meta-analysis of data from more than 1 million men and women. Lancet 2016, 388, 1302-1310. [CrossRef]

16. Czeisler, C.A. Impact of Sleepiness and Sleep Deficiency on Public Health-Utility of Biomarkers. J. Clin. Sleep Med. 2011, 7, S6-S8. [CrossRef] [PubMed]

17. Reiner, M.; Niermann, C.; Jekauc, D.; Woll, A. Long-term health benefits of physical activity-A systematic review of longitudinal studies. BMC Public Health 2013, 13, 813. [CrossRef] [PubMed]

18. Luyster, F.S.; Strollo, P.J.; Zee, P.C.; Walsh, J.K. Sleep: A Health Imperative. Sleep 2012, 35, 727-734. [CrossRef]

19. DeGroote, L.; Hamerlinck, G.; Poels, K.; Maher, C.; Crombez, G.; De Bourdeaudhuij, I.; VandenDriessche, A.; Curtis, R.G.; Desmet, A. Low-Cost Consumer-Based Trackers to Measure Physical Activity and Sleep Duration Among Adults in Free-Living Conditions: Validation Study. JMIR Mhealth Uhealth 2020. [CrossRef] [PubMed]

20. Dzierzewski, J.M.; Buman, M.P.; Giacobbi, P.R.; Roberts, B.L.; Aiken-Morgan, A.T.; Marsiske, M.; McCrae, C.S. Exercise and sleep in community-dwelling older adults: Evidence for a reciprocal relationship. J. Sleep Res. 2014, 23, 61-68. [CrossRef]

21. Mallinson, D.C.; Kamenetsky, M.E.; Hagen, E.W.; Peppard, P.E. Subjective sleep measurement: Comparing sleep diary to questionnaire. Nat. Sci. Sleep 2019, 11, 197-206. [CrossRef] [PubMed]

22. Buman, M.P.; King, A.C. Exercise as a Treatment to Enhance Sleep. Am. J. Lifestyle Med. 2010, 4, 500-514. [CrossRef]

23. Buman, M.P.; Winkler, E.A.H.; Kurka, J.M.; Hekler, E.B.; Baldwin, C.M.; Owen, N.; Ainsworth, B.E.; Healy, G.N.; Gardiner, P.A. Reallocating Time to Sleep, Sedentary Behaviors, or Active Behaviors: Associations With Cardiovascular Disease Risk Biomarkers, NHANES 2005-2006. Am. J. Epidemiol. 2014, 179, 323-334. [CrossRef]

24. Chastin, S.F.M.; Palarea-Albaladejo, J.; Dontje, M.L.; Skelton, D.A. Combined Effects of Time Spent in Physical Activity, Sedentary Behaviors and Sleep on Obesity and Cardio-Metabolic Health Markers: A Novel Compositional Data Analysis Approach. PLoS ONE 2015, 10. [CrossRef]

25. Cellini, N.; McDevitt, E.A.; Mednick, S.C.; Buman, M.P. Free-living cross-comparison of two wearable monitors for sleep and physical activity in healthy young adults. Physiol. Behav. 2016, 157, 79-86. [CrossRef] [PubMed]

26. Lymberis, A.; de Rossi, D. Wearable eHealth Systems for Personalised Health Management: State of the Art and Future Challenges; IOS Press: Amsterdam, The Netherlands, 2004

27. Hildebrand, M.; Van Hees, V.T.; Hansen, B.H.; Ekelund, U. Age Group Comparability of Raw Accelerometer Output from Wristand Hip-Worn Monitors. Med. Sci. Sports Exerc. 2014, 46, 1816-1824. [CrossRef] [PubMed]

28. Hildebrand, M.; Hansen, B.H.; Van Hees, V.T.; Ekelund, U. Evaluation of raw acceleration sedentary thresholds in children and adults. Scand. J. Med. Sci. Sports 2017, 27, 1814-1823. [CrossRef]

29. Aguilar-Farías, N.; Brown, W.J.; Peeters, G. ActiGraph GT3X+ cut-points for identifying sedentary behaviour in older adults in free-living environments. J. Sci. Med. Sport 2014, 17, 293-299. [CrossRef]

30. Van Hees, V.T.; Sabia, S.; Anderson, K.N.; Denton, S.J.; Oliver, J.; Catt, M.; Abell, J.G.; Kivimäki, M.; Trenell, M.I.; Singh-Manoux, A. A Novel, Open Access Method to Assess Sleep Duration Using a Wrist-Worn Accelerometer. PLoS ONE 2015, 10. [CrossRef]

31. Sadeh, A.; Sharkey, K.M.; Carskadon, M.A. Activity-Based Sleep-Wake Identification: An Empirical Test of Methodological Issues. Sleep 1994, 17, 201-207. [CrossRef] [PubMed] 
32. Kelly, J.M.; Strecker, R.E.; Bianchi, M.T. Recent Developments in Home Sleep-Monitoring Devices. ISRN Neurol. 2012, 2012, e768794. [CrossRef] [PubMed]

33. Westerterp, K.R. Physical activity and physical activity induced energy expenditure in humans: Measurement, determinants, and effects. Front. Physiol. 2013, 4, 90. [CrossRef] [PubMed]

34. Melanson, E.L.; Freedson, P.S.; Blair, S. Physical activity assessment: A review of methods. Crit. Rev. Food Sci. Nutr. 1996, 36, 385-396. [CrossRef]

35. Schoeller, D.A.; Jefford, G. Determinants of the energy costs of light activities: Inferences for interpreting doubly labeled water data. Int. J. Obes. 2002, 26. [CrossRef]

36. Neil-Sztramko, S.E.; Rafn, B.S.; Gotay, C.C.; Campbell, K.L. Determining activity count cut-points for measurement of physical activity using the Actiwatch2 accelerometer. Physiol. Behav. 2017, 173, 95-100. [CrossRef]

37. Lambiase, M.J.; Gabriel, K.P.; Kuller, L.H.; Matthews, K.A. Temporal Relationships between Physical Activity and Sleep in Older Women. Med. Sci. Sports Exerc. 2013, 45. [CrossRef] [PubMed]

38. Lambiase, M.J.; Gabriel, K.P.; Chang, Y.-F.; Kuller, L.H.; Matthews, K.A. Utility of Actiwatch Sleep Monitor to Assess Waking Movement Behavior in Older Women. Med. Sci. Sports Exerc. 2014, 46, 2301-2307. [CrossRef] [PubMed]

39. Lee, P.H.; Suen, L.K.P. The convergent validity of Actiwatch 2 and ActiGraph Link accelerometers in measuring total sleeping period, wake after sleep onset, and sleep efficiency in free-living condition. Sleep Breath. 2017, 21, 209-215. [CrossRef] [PubMed]

40. Quante, M.; Kaplan, E.R.; Rueschman, M.; Cailler, M.; Buxton, O.M.; Redline, S. Practical considerations in using accelerometers to assess physical activity, sedentary behavior, and sleep. Sleep Health 2015, 1, 275-284. [CrossRef]

41. Shin, M.; Swan, P.; Chow, C.M. The validity of Actiwatch2 and SenseWear armband compared against polysomnography at different ambient temperature conditions. Sleep Sci. 2015, 8, 9-15. [CrossRef]

42. Zhang, S.; Rowlands, A.V.; Murray, P.; Hurst, T.L. Physical Activity Classification Using the GENEA Wrist-Worn Accelerometer. Med. Sci. Sports Exerc. 2012, 44, 742-748. [CrossRef]

43. Lee, P.; Tse, C. Calibration of wrist-worn ActiWatch 2 and ActiGraph wGT3X for assessment of physical activity in young adults. Gait Posture 2019, 68, 141-149. [CrossRef]

44. Meltzer, L.J.; Walsh, C.M.; Traylor, J.; Westin, A.M.L. Direct comparison of two new actigraphs and pol-ysomnography in children and adolescents. Sleep 2012, 35, 159-166.

45. Leinonen, A.-M.; Ahola, R.; Kulmala, J.; Hakonen, H.; Vähä-Ypyä, H.; Herzig, K.-H.; Auvinen, J.; Keinänen-Kiukaanniemi, S.; Sievänen, H.; Tammelin, T.H.; et al. Measuring Physical Activity in Free-Living Conditions—Comparison of Three AccelerometryBased Methods. Front. Physiol. 2017, 7. [CrossRef] [PubMed]

46. Troiano, R.P.; McClain, J.J.; Brychta, R.J.; Chen, K.Y. Evolution of accelerometer methods for physical activity research. Br. J. Sports Med. 2014, 48, 1019-1023. [CrossRef] [PubMed]

47. Cheung, J.; Zeitzer, J.M.; Lu, H.; Mignot, E. Validation of minute-to-minute scoring for sleep and wake periods in a consumer wearable device compared to an actigraphy device. Sleep Sci. Pract. 2018, 2, 11. [CrossRef]

48. Esliger, D.W.; Rowlands, A.V.; Hurst, T.L.; Catt, M.; Murray, P.; Eston, R.G. Validation of the GENEA Accelerometer. Med. Sci. Sports Exerc. 2011, 43, 1085-1093. [CrossRef]

49. Marschollek, M.; Gietzelt, M.; Schulze, M.; Kohlmann, M.; Song, B.; Wolf, K.-H. Wearable Sensors in Healthcare and SensorEnhanced Health Information Systems: All Our Tomorrows? Healthc. Inform. Res. 2012, 18, 97-104. [CrossRef] [PubMed]

50. Baandrup, L.; Fasmer, O.B.; Glenthøj, B.Y.; Jennum, P.J. Circadian rest-activity rhythms during benzodiazepine tapering covered by melatonin versus placebo add-on: Data derived from a randomized clinical trial. BMC Psychiatry 2016, 16, 348. [CrossRef]

51. Gresham, G.; Hendifar, A.E.; Spiegel, B.; Neeman, E.; Tuli, R.; Rimel, B.J.; Figlin, R.A.; Meinert, C.L.; Piantadosi, S.; Shinde, A.M. Wearable activity monitors to assess performance status and predict clinical outcomes in advanced cancer patients. NPJ Digit. Med. 2018, 1. [CrossRef]

52. Tang, W.; Sazonov, E.S. Highly Accurate Recognition of Human Postures and Activities through Classification with Rejection. IEEE J. Biomed. Health Inform. 2014, 18, 309-315. [CrossRef]

53. Li, P.; Wang, Y.; Tian, Y.; Zhou, T.-S.; Li, J.-S. An Automatic User-adapted Physical Activity Classification Method Using Smartphones. IEEE Trans. Biomed. Eng. 2016, 64, 706-714. [CrossRef]

54. Migueles, J.H.; Cadenas-Sanchez, C.; Ekelund, U.; Nyström, C.D.; Mora-Gonzalez, J.; Löf, M.; Labayen, I.; Ruiz, J.R.; Ortega, F.B. Accelerometer Data Collection and Processing Criteria to Assess Physical Activity and Other Outcomes: A Systematic Review and Practical Considerations. Sports Med. 2017, 47, 1821-1845. [CrossRef] [PubMed]

55. Smith, C.; Galland, B.; Taylor, R.; Meredith-Jones, K. ActiGraph GT3X+ and Actical Wrist and Hip Worn Accelerometers for Sleep and Wake Indices in Young Children Using an Automated Algorithm: Validation With Polysomnography. Front. Psychiatry 2020, 10. [CrossRef] [PubMed]

56. Moore, L.; McDevitt, B.; Akhtar, N.; Doherty, R.; Scott, W.N.; Connolly, J. Preliminary investigations of the agreement between two wearable accelerometers for use in clinical studies. In Proceedings of the 31st Irish Signals and Systems Conference (ISSC 2020), Letterkenny, Ireland, 11-12 June 2020; pp. 1-4. [CrossRef]

57. Philips Healthcare. Actiwatch 2 Activity Monitor. 2020. Available online: https://www.usa.philips.com/healthcare/product/ HC1044809/actiwatch-2-activity-monitor (accessed on 3 December 2020). 
58. Born, A. Verisense IMU Supports Clinical Trials as CE Certified Class 1 Medical Device. Electronics360. 2020. Available online: https:/ / electronics360.globalspec.com/article/16100/verisense-imu-supports-clinical-trials-as-ce-certified-class-1-medicaldevice?from_rss=1 (accessed on 3 December 2020).

59. Shimmer Research Ltd. IMU Specifications. 2020. Available online: https://static1.squarespace.com/static/5da5da507123036dd4 7fac05/t/5df8f77d2969387e13f7918a/1576597398078/Spec+sheet.pdf (accessed on 30 December 2020).

60. SleepFoundation.org, “Sample Sleep Log," SleepFoundation.org. 2020. Available online: https://www.sleepfoundation.org/ sample-sleep-log (accessed on 30 December 2020).

61. Cambridge Biomedical Research Centre. DAPA Measurement Toolkit. 2020. Available online: https://dapa-toolkit.mrc.ac.uk/ physical-activity/subjective-methods/diaries-and-logs (accessed on 30 December 2020).

62. Matthews, C.E.; Ainsworth, B.E.; Hanby, C.; Pate, R.R.; Addy, C.; Freedson, P.S.; Jones, D.A.; Macera, C.A. Development and testing of a short physical activity recall questionnaire. Med. Sci. Sports Exerc. 2005, 37, 986-994. [PubMed]

63. Van Hees, V.T.; Sabia, S.; Jones, S.E.; Wood, A.R.; Anderson, K.N.; Kivimäki, M.; Frayling, T.M.; Pack, A.I.; Bucan, M.; Trenell, M.I.; et al. Estimating sleep parameters using an accelerometer without sleep diary. Sci. Rep. 2018, 8, 12975. [CrossRef]

64. Shimmer Research Ltd. About, Verisense. 26 June 2019. Available online: https://www.verisense.net/about (accessed on 5 January 2020).

65. Philips Healthcare. Actiware; Philips Healthcare: Andover, MA, USA, 2020.

66. Welk, G.J. Principles of Design and Analyses for the Calibration of Accelerometry-Based Activity Monitors. Med. Sci. Sports Exerc. 2005, 37, S501. [CrossRef]

67. Bassett, D.R.; Freedson, P.; Kozey, S. Medical Hazards of Prolonged Sitting. Exerc. Sport Sci. Rev. 2010, 38, 101-102. [CrossRef]

68. Van Hees, V.T.; Gorzelniak, L.; León, E.C.D.; Eder, M.; Pias, M.; Taherian, S.; Ekelund, U.; Renström, F.; Franks, P.W.; Horsch, A.; et al. Separating Movement and Gravity Components in an Acceleration Signal and Implications for the Assessment of Human Daily Physical Activity. PLoS ONE 2013, 8, e61691. [CrossRef]

69. Van Hees, V.T.; Fang, Z.; Langford, J.; Assah, F.; Mohammad, A.; Da Silva, I.C.M.; Trenell, M.I.; White, T.; Wareham, N.J.; Brage, $\mathrm{S}$. Autocalibration of accelerometer data for free-living physical activity assessment using local gravity and temperature: An evaluation on four continents. J. Appl. Physiol. 2014, 117, 738-744. [CrossRef]

70. R Core Team. The R Project for Statistical Computing; R Core Team: Vienna, Austria, 2020.

71. Migueles, J.H.; Rowlands, A.V.; Huber, F.; Sabia, S.; Van Hees, V.T. GGIR: A Research Community-Driven Open Source R Package for Generating Physical Activity and Sleep Outcomes From Multi-Day Raw Accelerometer Data. J. Meas. Phys. Behav. 2019, 2, 188-196. [CrossRef]

72. Sabia, S.; Van Hees, V.T.; Shipley, M.J.; Trenell, M.I.; Hagger-Johnson, G.; Elbaz, A.; Kivimaki, M.; Singh-Manoux, A. Association Between Questionnaire- and Accelerometer-Assessed Physical Activity: The Role of Sociodemographic Factors. Am. J. Epidemiol. 2014, 179, 781-790. [CrossRef]

73. IBM Corp. IBM SPSS Statistics for Windows, Version 26.0; IBM Corp: Armonk, NY, USA, 2019.

74. Lehman, A. JMP for Basic Univariate and Multivariate Statistics: A Step-by-Step Guide; SAS Press: Cary, NC, USA, 2005.

75. Swinscow, T.D.V. "Statistics at Square One," The BMJ I The BMJ: Leading General Medical Journal. Research. Education. Comment. 1997. Available online: https:/ / www.bmj.com/about-bmj/resources-readers/publications/statistics-square-one (accessed on 30 December 2020).

76. Mukaka, M.M. A guide to appropriate use of Correlation coefficient in medical research. Malawi Med. J. 2012, 24, 69-71. [PubMed]

77. Šimundić, A.-M. Measures of Diagnostic Accuracy: Basic Definitions. EJIFCC 2009, 19, 203-211.

78. Giavarina, D. Understanding Bland Altman analysis. Biochem. Med. 2015, 25, 141-151. [CrossRef]

79. Chin, S. Open-Source Initiative Paving Way for Wearable Sensor Standards. FierceElectronics. 16 September 2019. Available online: https:/ / www.fierceelectronics.com/sensors/open-source-initiative-paving-way-for-wearable-sensor-standards (accessed on 8 October 2020).

80. Spinner, J. Wearables Group Seeks Collaboration on Decentralized Tech. 11 May 2020. Available online: https:/ /www.outsourcingpharma.com/Article/2020/11/05/Wearables-group-seeks-collaboration-on-decentralized-tech (accessed on 30 December 2020).

81. OWEAR. Open Source Algorithms \& Data Sets. Wearable Technology Software Database. Available online: https://www.owear. org (accessed on 30 December 2020).

82. Welch, W.A.; Bassett, D.R.; Thompson, D.L.; Freedson, P.S.; Staudenmayer, J.W.; John, D.; Steeves, J.A.; Conger, S.A.; Ceaser, T.; Howe, C.A.; et al. Classification Accuracy of the Wrist-Worn GENEA Accelerometer. Med. Sci. Sports Exerc. 2013, 45, 2012-2019. [CrossRef]

83. Loprinzi, P.D.; Smith, B. Comparison between Wrist-Worn and Waist-Worn Accelerometry. J. Phys. Act. Health 2017, 14, 539-545. [CrossRef]

84. Soaz, C.; Diepold, K. Step Detection and Parameterization for Gait Assessment Using a Single Waist-Worn Accelerometer. IEEE Trans. Biomed. Eng. 2016, 63, 933-942. [CrossRef] [PubMed]

85. Wolff-Hughes, D.L.; Bassett, D.R.; Fitzhugh, E.C. Population-Referenced Percentiles for Waist-Worn Accelerometer-Derived Total Activity Counts in U.S. Youth: 2003-2006 NHANES. PLoS ONE 2014, 9, e115915. [CrossRef] [PubMed]

86. National Center for Health Statistics (U.S.) (Ed.) National Health and Nutrition Examination Survey. National Youth Fitness Survey Plan, Operations, and Analysis, 2012; U.S. Department of Health and Human Services, Centers for Disease Control and Prevention, National Center for Health Statistics: Hyattsville, MD, USA, 2014.

87. Swartz, A.M.; Strath, S.J.; Bassett, D.R.; O’Brien, W.L.; King, G.A.; Ainsworth, B.E. Estimation of energy expenditure using CSA accelerometers at hip and wrist sites. Med. Sci. Sports Exerc. 2000, 32, S450-S456. [CrossRef] [PubMed] 